\title{
Image Segmentation Using Ant System-based Clustering Algorithm
}

\author{
Aleksandar Jevtić, Joel Quintanilla-Domínguez, José Miguel Barrón-Adame and \\ Diego Andina
}

\begin{abstract}
Industrial applications of computer vision sometimes require detection of atypical objects that occur as small groups of pixels in digital images. These objects are difficult to single out because they are small and randomly distributed. In this work we propose an image segmentation method using the novel Ant System-based Clustering Algorithm (ASCA). ASCA models the foraging behaviour of ants, which move through the data space searching for high data-density regions, and leave pheromone trails on their path. The pheromone map is used to identify the exact number of clusters, and assign the pixels to these clusters using the pheromone gradient. We applied ASCA to detection of microcalcifications in digital mammograms and compared its performance with state-of-the-art clustering algorithms such as 1D Self-Organizing Map, $k$-Means, Fuzzy $c$-Means and Possibilistic Fuzzy $c$-Means. The main advantage of ASCA is that the number of clusters needs not to be known a priori. The experimental results show that ASCA is more efficient than the other algorithms in detecting small clusters of atypical data.
\end{abstract}

\section{Introduction}

Soft-computing methods have been widely used in industrial and environmental applications. They usually find application to complex optimization problems for which approximate solutions are acceptable within a given error margin. Softcomputing methods have been successfully applied to knowledge extraction [1],

Aleksandar Jevtić, Joel Quintanilla-Domínguez and Diego Andina

E.T.S.I. de Telecomunicación, Universidad Politécnica de Madrid, Avda. Complutense 30, Madrid 28040, Spain.e-mail: aleksandar.jevtic@upm.es; joelq@ salamanca.ugto.mx; d.andina@upm.es

José Miguel Barrón Adame

División de Ingenierias, Campus Irapuato-Salamanca, Universidad de Guanajuato, Carretera Salamanca-Valle de Santiago Km $3.5+1.8 \mathrm{Km}$ Comunidad de Palo Blanco, Salamanca, Gto., 36885, Mexico. e-mail: badamem@ salamanca.ugto.mx 
information security [2], manufacturing [3], sustainable building [4], meteorology [5], etc.

In applications of computer vision, image segmentation is an important preprocessing step. The objective is to partition the image into homogeneous regions that share certain visual characteristics. There is therefore a natural tendency to apply data clustering algorithms to image segmentation. Clustering is a method of unsupervised learning because no prior labeling of data is available [6].

In computer vision applications such as digital mammography for cancer risk analysis, the less representative pixels are the most interesting since they represent a variation with respect to healthy tissue. Pixels of high grey-level intensity may indicate the presence of microcalcifications which may be an early sign of breast cancer. These microcalcifications are difficult to detect because they appear in groups of just a few pixels, which are often merged with larger clusters of high intensity in their proximity.

For the image segmentation method, the ASCA is proposed to group the pixels of similar grey-level intensity. The proposed method is based on the Ant System (AS) algorithm, which was originally created to solve the traveling salesman problem [7]. The AS models the pheromone-laying behaviour of ants for a discrete data space, which in our case is a digital image. For the experiments, two types of images were used. In these pictures, imperfections can be the result of light reflection over the image, or real imperfections that can be used as an aid in medical diagnosis. ASCA uses only the intensity level of the grey-level images as a criterion for image segmentation.

The paper is organised as follows. Sect. 2 provides a summary of related literature. Sect. 3 describes the proposed ASCA algorithm. The image segmentation method based on the proposed algorithm is presented together with the experimental results in Sect. 4. The results are discussed and compared with those obtained by the current state-of-the-art clustering algorithms. Finally, in Sect. 5 the conclusions are drawn.

\section{Related Work}

In this section, we give an overview of the state-of-the-art clustering algorithms. For image segmentation, clustering can be considered a preprocessing step that does not include the spatial information of the pixels. Therefore, the goal of the clustering process is to group the pixels based on their similarities in order to facilitate further knowledge extraction. Kotsiantis and Pintelas [8] define the following five categories of clustering algorithms: partitioning methods, hierarchical methods, density-based methods, grid-based methods and model-based methods. The swarmbased algorithms do not explicitly belong to any of the named categories [9].

The Self-Organizing Maps (SOM) [10], an Artificial Neural Network (ANN) with unsupervised learning, is a widely used clustering algorithm. SOM is useful for data classification because of its visualization property. It was applied to pattern 
recognition in satellite images [11], segmentation of colour images [12], but also many others.

A cluster of pixels is usually associated with a prototype as the most representative pixel also considered the cluster centre. Some clustering methods use this centric property to divide $N$-dimensional data space, where the partitions are either strict, fuzzy or possibilistic. A well-known strict partitioning algorithm is the $k$-Means [13] which divides a data set into $k$ subsets such that all points in a given subset are closest to the same centre. In order to obtain more information about the proximity of a data point to a prototype, Bezdek [14] proposed the Fuzzy $c$-Means algorithm (FCM) that calculates a membership degree for each data point in relation to different clusters. Since several equidistant data with the same membership values are not equally representative of the clusters, the Possibilistic $c$-Means algorithm (PCM) algorithm was proposed in [15], which identified the similarity of data with a given number of prototypes using typicality values ranging from $(0$, 1). Pal et al. proposed to use both membership degrees and typicality values and implemented it in the Fuzzy Possibilistic $c$-Means (FPCM) algorithm [16]. Another improvement was proposed with the Possibilistic Fuzzy $c$-Means (PFCM) algorithm [17] by introducing the control parameters which define the relative importance of the membership degrees and the typicality values.

The clustering performance of the mentioned algorithms is greatly dependent on the initial guess of cluster centres and it is time consuming. Various methods were proposed to address these issues. One simple implementation of the $k$-Means algorithm to colour quantization, data compression and image segmentation was proposed in [18]. The algorithm stores the multidimensional data points in a $k d$-tree that is computed only once, which results in faster computation. Laia and Liaw [19] proposed a modified $k$-Means algorithm to speed up the clustering process for larger data sets with higher dimension. Some clustering methods were proposed to reduce the effect of the initially selected cluster centres [20] or for a more robust and less noise-sensitive clustering [21].

The sensitivity in detection of the atypical data remains an issue for the state-ofthe-art clustering algorithms. Ojeda et al. [22] propose an image sub-segmentation method based on the PFCM algorithm in order to detect small homogeneous regions in mammograms. The authors applied a typicality value threshold to delimit a subgroup containing atypical pixels within the initially detected clusters. The threshold value was set manually which is the main drawback of this method.

Ant clustering algorithms are inspired by the swarming behaviour of natural ant colonies. Handl and Meyer [9] roughly classify these algorithms in two main groups. The first group mimics the corpse gathering and brood sorting in natural ant colonies, where the clustering solution emerges as a result of interactions of ants with the environment. The second group of algorithms uses general-purpose antbased optimization methods in order to obtain optimal clustering of the given data set. There are also algorithms that do not explicitly belong to any of these groups, but fall somewhere in between. 


\section{Ant System-based Clustering Algorithm}

The novel Ant System-based Clustering Algorithm (ASCA) proposed in this paper was inspired by the foraging behaviour of ant colonies in nature. When ants find a food source, they leave pheromone trails that attract other ants to follow their path. Pheromone trails evaporate over time, so a path that leads to a closer food source accumulates more pheromone as it is crossed by ants more frequently. The AS algorithm exploits this cooperative behaviour of ant colonies that features indirect communication through the environment. Unlike their biological counterparts, the artificial ants move through a discrete space defined with nodes and they have memory of the taken path.

Pheromone trails in the ASCA algorithm are accumulated in nodes in order to represent the density of the surrounding data. This differs from what was proposed in basic Ant System algorithm where pheromone trails marked the edges that connected the nodes in order to represent the favorite path. The process of pheromone accumulation is iterative and creates a pheromone map of the data set we want to cluster. Higher data-density areas accumulate more pheromone and they represent cluster centres. This is used to extract the number of clusters. Gradient of the pheromone trail is used to assign every node to a cluster by applying local Hill-climbing search [23]. The ASCA algorithm consists of three consecutive parts, namely: a) pheromone accumulation, b) local pheromone summing, and c) data labeling.

\subsection{Pheromone Accumulation}

In the pheromone accumulation stage, the artificial ants move in $\mathrm{N}$-dimensional data space looking for the high data-density regions. The algorithm starts with an initialization step which is followed by the iterative construction of new solutions and pheromone update. It involves the following steps:

1. Initialization: All nodes are initialised with an equal small amount of pheromone, $\tau_{0}$. The population of $M$ ants is created and placed on randomly chosen nodes.

2. Node transition rule: Ant chooses the next node to move to by applying the roulette rule. That is, every node has associated probability with which it is chosen from a set of available nodes. The probability of displacing $k$ th ant from node $i$ to node $j$ depends on the Euclidean distance between the nodes and the amount of pheromone trail accumulated in node $j$, and it is given by:

$$
p_{i j}^{k}= \begin{cases}\frac{\left(\tau_{j}\right)^{\alpha}\left(\eta_{i j}\right)^{\beta}}{\sum_{h \notin t a b u_{k}},\left(\tau_{h}\right)^{\alpha}\left(\eta_{i h}\right)^{\beta}}, & \text { if } j \notin t a b u_{k} \\ 0, & \text { otherwise }\end{cases}
$$


where $\tau_{j}$ and $\eta_{i j}$ are the intensity of the pheromone trail on the node $j$ and the visibility of the node $j$ with respect to the node $i$, respectively. The visibility is given as the reciprocal value of the Euclidean distance, $d_{i j}$, between the nodes $i$ and $j: \eta_{i j}=1 / d_{i j}$. Ant is not allowed to displace to the nodes it has already visited. $T a b u_{k}$ list contains the nodes visited by the $k$ th ant. The control parameters $\alpha$ and $\beta$ allow us to bias the decision-making mechanism towards the exploitation of the generated knowledge about the environment or exploration of new solutions, respectively. ( $\alpha, \beta>0 ; \alpha, \beta \in \Re$.) It can be noticed that the accumulated pheromone trails serve as a colony's external memory where the extracted knowledge about the environment is stored.

3. Pheromone update rule: Once all the ants carry out the transition to other nodes, the pheromone update is applied to each node as follows:

$$
\tau_{j, \text { new }}=(1-\rho) \tau_{j, \text { old }}+\sum_{k=1}^{M} \Delta \tau_{j}^{k}
$$

where $\rho$ is the pheromone evaporation rate $(0<\rho<1 ; \rho \in \Re)$, and $\Delta \tau_{j}^{k}$ is the amount of pheromone laid on the node $j$ by the $k$ th ant, and is given by:

$$
\Delta \tau_{j}^{k}=\left\{\begin{array}{l}
\eta_{i j}, \text { if node } j \text { has been visited by } k \text { th ant } \\
0, \quad \text { otherwise. }
\end{array}\right.
$$

where $\eta_{i j}$ is the visibility of the node $j$ from the node $i$ from which the $k$ th ant was displaced.

4. Stopping criterion: The steps 2 and 3 are repeated in a loop and the algorithm stops executing when the maximum number of iterations is reached.

The output is a pheromone matrix where the distribution of pheromone is scarce, but the highest concentrations are found around the dense regions of nodes in data space. The ants make a probabilistic choice of path, therefore the neighbouring nodes may have significantly different amounts of pheromone deposits (see Fig. 1(b)). For this reason, we apply the local pheromone summing.

\subsection{Local Pheromone Summing}

The pheromone trails are locally summed to obtain a smooth pheromone surface to which a local gradient-based search will be applied. We define the neighbourhood resolution as an $\mathrm{N}$-dimensional sphere that represents a portion of the Euclidean data space, and whose diameter is given by:

$$
r=\frac{1}{\gamma} \sqrt{\sum_{n=1}^{N}\left(x_{n, \max }-x_{n, \min }\right)^{2}}
$$




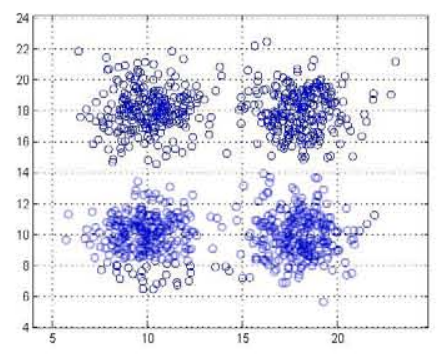

(a) Squares data set

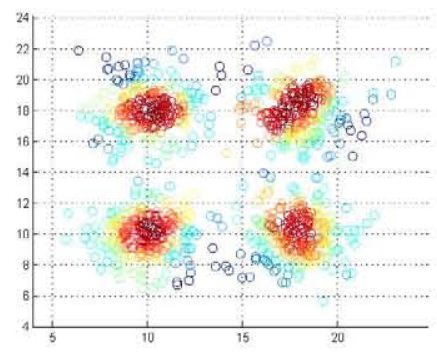

(c) After pheromone summing

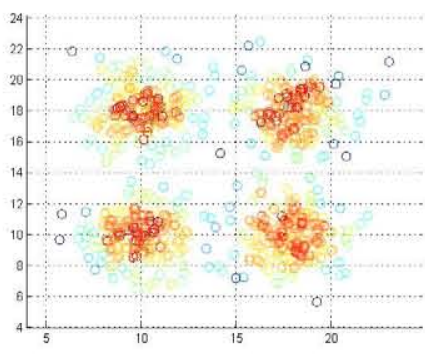

(b) After pheromone accumulation

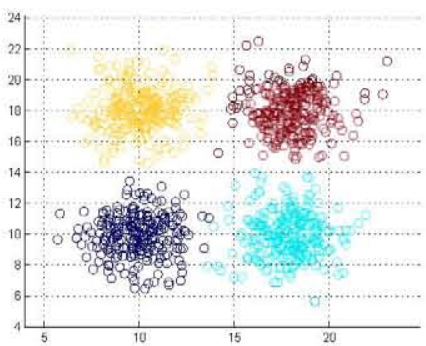

(d) After data labeling

Fig. 1 Clustering result after applying the proposed ASCA algorithm to a Squares data set, which consists of four groups of 250 data patterns with normal Gaussian distribution.

where $\gamma$ is the resolution ratio, and $\left(x_{n, \min }, x_{n, \max }\right)$ is the data space range along the $n$th dimension. For each node $i$, the pheromone deposits from the neighbouring nodes are added to already present pheromone value, $\tau_{i 0}$, as follows:

$$
\tau_{i s}=\tau_{i 0}+\sum_{n=1}^{N_{\text {neigh }}} \tau_{n}
$$

where $N_{\text {neigh }}$ is the number of neighbouring nodes contained within the sphere defined in Eq. 4.

The output of the local pheromone summing process is a smooth $(N+1)$ dimensional surface with pheromone value peaks around the cluster centres (see Fig. 1(c)).

\subsection{Data Labeling}

In the final step of the ASCA algorithm, the discrete Hill-climbing gradient-based search method is applied to find the local maxima on the pheromone surface. The 
search is performed from each node with the step size defined in Eq. 4. The nodes which lead to the same local maximum are grouped in one cluster (see Fig. 1(d)). The number of clusters is therefore equal to the number of local maxima. It is important to emphasise that the ASCA algorithm extracts the number of clusters from the data set, unlike the other algorithms compared that require it to be set in advance.

\section{Experimental Results and Discussion}

In this section the ASCA algorithm is applied to the image segmentation task. The proposed method mimics the distributed organization of an artificial ant colony, and exploits the pheromone patterns that emerge as a result of local interactions. Specifically, the algorithm creates the pheromone mapping of a set of unlabeled image pixels in order to cluster them according to their grey-scale intensity level. The objective is to detect the atypical pixels, and for this two kinds of images are used, the "Splash" image, see Fig. 2(a), and a mammogram, see Fig. 3(a).

For all the images, the parameters of the algorithm are set to: $\tau_{0}=100, \alpha=1$, $\beta=5, \rho=0.05, \gamma=30$. The experiments were performed in 3 cycles with 30 iterations. In each cycle, a population of 1000 ants were displaced on randomly chosen nodes. The experiments were performed using MATLAB (software MATLAB, version R2009b), on a computer with Pentium IV processor at $3.4 \mathrm{GHz}$, with $2 \mathrm{~GB}$ of RAM. For the $k$-Means and FCM algorithms, the native MATLAB functions were used. The settings for the 1D-SOM and the PFCM were implemented as proposed in [24] and [22], respectively. The results of experiments are shown in Fig. 2, for the "Splash" image, and Fig. 3 for a region of interest (ROI) mammogram.

For the "Splash" image the goal was to detect the pixels of high grey-level intensity that are a result of light reflection. The ASCA algorithm extracted six clusters which was enough to single out the atypical pixels. A comparison was made with 1D-SOM, $k$-Means and FCM algorithms using the same number of clusters. The ASCA outperformed 1D-SOM, $k$-Means and FCM. None of the control algorithms was able to extract the regions of interest. In case of the PFCM, image sub-segmentation was applied after the initial segmentation in two pixel groups. Because of the limitation of this approach to divide the data space in $2^{n}$ partitions, the segmentation was performed for four clusters as proposed by the authors. Although the PFCM managed to detect the light reflection pixels, some other features like the shadow of the splash were not extracted. As a second part of the experiment, though not presented here, for each algorithm we incremented the number of clusters until the image segmentation allowed the separation of light reflection pixels. We obtained the following results: 1D-SOM, 17 clusters; $k$-Means, 9 clusters; and FCM, 7 clusters.

In mammography, the goal is to detect the presence of microcalcifications that could be an early sign of breast cancer. They appear as small groups of pixels of high grey-level intensity and they usually occupy a very small range of values, hence they are hard to detect. The ROI mammograms on which the experiments 


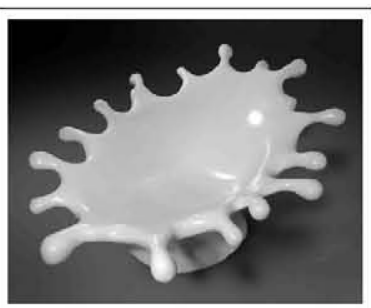

(a) Original image

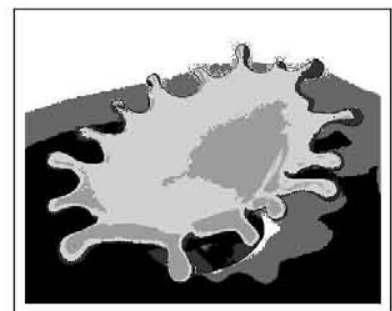

(d) FCM

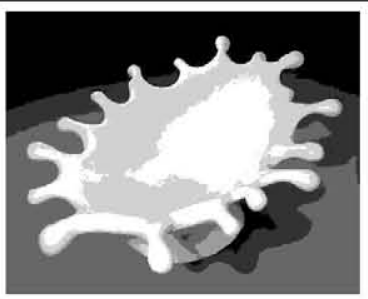

(b) 1D-SOM

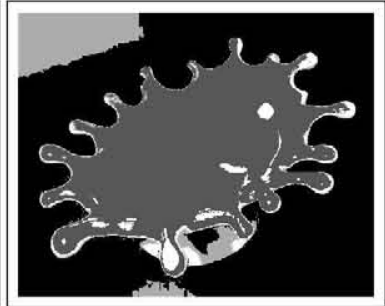

(e) PFCM sub-segm.

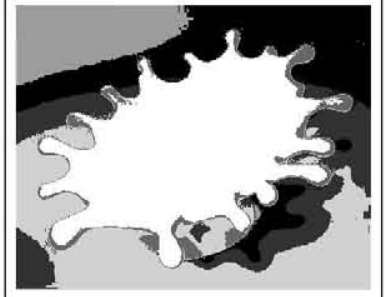

(c) $k$-Means

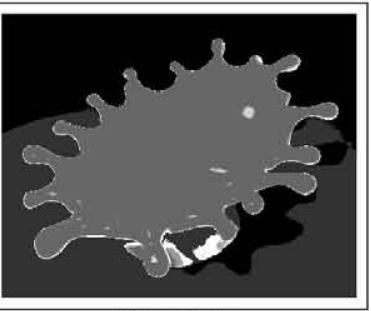

(f) ASCA

Fig. 2 Comparison of the segmentation results for the "Splash" image, $320 \times 400$ pixels. The ASCA extracted six clusters; for the purpose of comparison, other algorithms were set to partition the data set in six clusters as well, only in case of PFCM with sub-segmentation the results are shown for four clusters because of the limitation of the algorithm to have $2^{n}$ partitions.

were performed and the results of image segmentation using 1D-SOM, $k$-Means, FCM, PFCM and the proposed ASCA are shown in Fig. 3. The ASCA extracted three clusters. For comparison, the same number of clusters was used for the segmentation trials based on the 1D-SOM, $k$-Means and FCM methods. The image segmentation using the latter three algorithms gave poor results as the microcalcification pixels could not be isolated. PFCM managed to detect the microcalcifications but with a higher number of clusters (four) than ASCA. Even increasing the number of clusters, 1D-SOM, $k$-Means and FCM were not able to obtain better segmentation results. The output images became over-segmented which prevented the extraction of the regions of interest.

For the ASCA algorithm, image pixels clustering comes as a result of the adaptive behaviour of the artificial ant colony. The ants find paths from the peripheral regions of a cluster to its centre, and accumulate there higher concentration of pheromone. The indirect interaction between the ants via the environment gives different results from what would be obtained by simple data-density calculation. Not all high-density regions accumulate equal amounts of pheromone even though their local neighbourhood may be similar (e.g. same distance from the nearest neighbour). The cluster centres are crossed by more ants from various directions, accumulating thus more pheromone than the peripheral regions. 


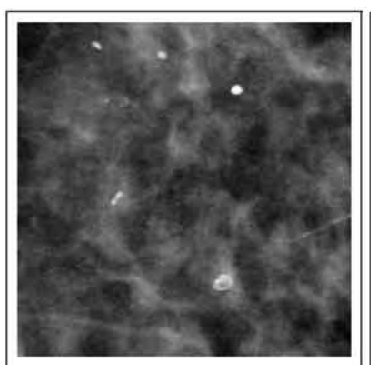

(a) Original image

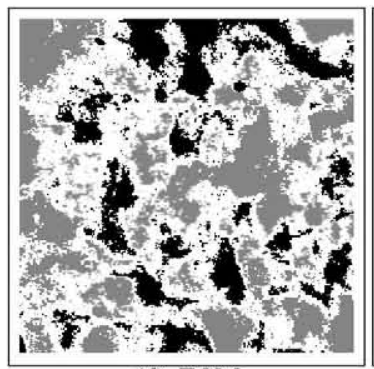

(d) FCM

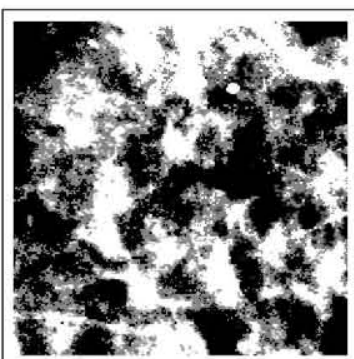

(b) 1D-SOM

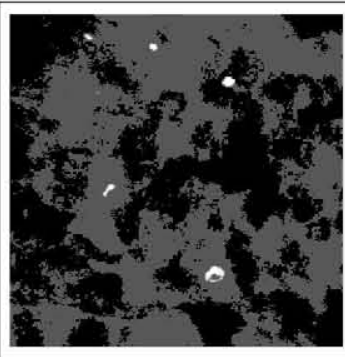

(e) PFCM sub-segm.

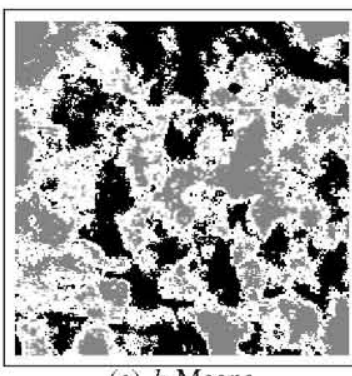

(c) $k$-Means

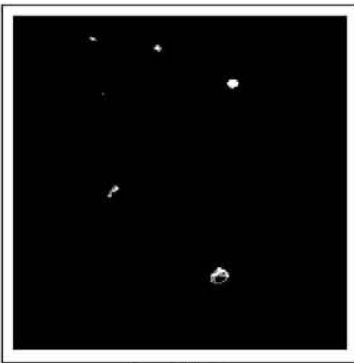

(f) ASCA

Fig. 3 Comparison of the segmentation results for a ROI mammogram, $256 \times 256$ pixels. The ASCA extracted three clusters; for the purpose of comparison, other algorithms were set to partition the data set in three clusters as well, only in case of PFCM with sub-segmentation the results are shown for four clusters because of the limitation of the algorithm to have $2^{n}$ partitions.

\section{Conclusions}

The important feature of the proposed ASCA algorithm is the automatic extraction of the number of clusters. This is useful for applications where the groups of patterns within a data set are not well-defined and need to be detected. The performance of the ASCA algorithm was evaluated through experiments on two types of images. The proposed method outperformed 1D-SOM, $k$-Means, FCM and PFCM algorithms in the detection of small, atypical regions of the image, in the overall quality of the segmented images, and in terms of the total number of clusters used. The resolution ratio $\gamma$ is used to set the sensitivity of the algorithm to the density of the data. Future work will include the optimization of the algorithm's parameters, namely the resolution ratio $\gamma$ and control parameters $\alpha$ and $\beta$, in order to improve the adaptability of the algorithm to different data sets. For the task of image processing, more features such as spatial information or texture will be used to obtain better segmentation results. Also, testing the algorithm on the colour images may open a wide range of new applications. 


\section{References}

1. Yu W-D, Liu Y-C (2006) Hybridization of CBR and numeric soft computing techniques for mining of scarce construction databases. Automat Constr 15(1):33-46

2. Corchado E, Herrero A (2010) Neural visualization of network traffic data for intrusion detection. Appl Soft Comput, doi: 10.1016/j.asoc.2010.07.002

3. Andina D, Pham D T (2007) Computational intelligence: For engineering and manufacturing. Springer-Verlag New York, Inc., Secaucus, NJ, USA

4. Sedano J, Curiel L, Corchado E, de la Cal E, Villar J R (2010) A soft computing method for detecting lifetime building thermal insulation failures. Integr Comput-Aided E 17(2):103-115

5. Corchado E, Arroyo A, Tricio V (2010) Soft computing models to identify typical meteorological days. Log J IGPL, doi:10.1093/jigpal/jzq035

6. Jain A K, Murty M N, Flynn P J (1999) Data clustering: A review. ACM Comput Surv 31(3):264-323

7. Dorigo M, Maniezzo V, Colorni A (1996) Ant System: Optimization by a colony of cooperating agents. IEEE T Syst Man Cyb - Part B 26(1):29-41

8. Kotsiantis S, Pintelas P (2004) Recent advances in clustering: A brief survey. WSEAS Transactions on Information Science and Applications 1(1):73-81

9. Handl J, Meyer B (2007) Ant-based and swarm-based clustering. Swarm Intelligence 1(1):95-113

10. Kohonen T (1990) The self-organizing map. P IEEE 78(9):1464-1480

11. Richardson A J, Risien C, Shillington F A (2003) Using self-organizing maps to identify patterns in satellite imagery. Prog Oceanogr 59:223-239

12. Jiang Y, Zhou Z H (2004) SOM ensemble-based image segmentation. Neural Process Lett 20:171-178

13. MacQueen J B (1967) Some methods for classification and analysis of multivariate observations. Proc $5^{t h}$ Berkeley Symposium on Mathematical Statistics and Probability, Berkeley, University of California Press 281-297

14. Bezdek J C (1981) Pattern recognition with fuzzy objective function algorithms. P. Press, edn. Plenum Press, New York

15. Krishnapuram R, Keller J M (1993) A possibilistic approach to clustering. IEEE T Fuzzy Syst 1(2): $98-110$

16. Pal N R, Pal K, Bezdek J C (1997) A mixed c-means clustering model. Proc $6^{\text {th }}$ IEEE Int Conf Fuzzy Syst 11-21

17. Pal N R, Pal K, Keller J M, and Bezdek, J C (2005) A possibilitic fuzzy c-means clustering algorithm. IEEE T Fuzzy Syst 13(4):517-530

18. Kanungo T, Mount D M, Netanyahu N S, Piatko C D, Silverman R, Wu A Y (2002) An efficient k-Means clustering algorithm: Analysis and implementation. IEEE T Pattern Anal 24(7):881-892

19. Laia J Z C, Liaw Y C (2008) Improvement of the k-means clustering filtering algorithm. Pattern Recogn 41:3677-3681

20. Chang K C, Yeh M F (2005) Grey relational analysis based approach for data clustering. IEE P-Vis Image Sign 152(2):165-172

21. Awad M, Chehdi K, Nasri A (2009) Multi-component image segmentation using a hybrid dynamic genetic algorithm and fuzzy C-means. IET Image Process 3(2):52-62

22. Ojeda-Magaña B, Quintanilla-Domínguez J, Ruelas R, Andina D (2009) Images subsegmentation with the PFCM clustering algorithm. Proc $7^{\text {th }}$ IEEE Int Conf Industrial Informatics $499-503$

23. Russell S J, Norvig P (2003) Artificial intelligence: A modern approach, 2nd edn. Prentice Hall, Upper Saddle River, New Jersey

24. Barrón-Adame J M, Herrera-Delgado J A, Cortina-Januchs M G, Andina D, Vega-Corona A (2007) Air pollutant level estimation applying a self-organizing neural network. Proc $2^{\text {nd }} \mathrm{Int}$ Work-conf Nature Inspired Problem-Solving Methods in Knowledge Engineering, Springer Berlin / Heidelberg, LNCS 4528:599-607 